DOI: $10.17516 / 1997-1397-2021-14-3-326-343$

УДК 517.55

\title{
On Transcendental Systems of Equations
}

\author{
Alexander M. Kytmanov* \\ Olga V.Khodos ${ }^{\dagger}$ \\ Siberian Federal University \\ Krasnoyarsk, Russian Federation
}

Received 10.12.2020, received in revised form 22.01.2021, accepted 20.03.2021

\begin{abstract}
Several types of transcendental systems of equations are considered: the simplest ones, special, and general. Since the number of roots of such systems, as a rule, is infinite, it is necessary to study power sums of the roots of negative degree. Formulas for finding residue integrals, their relation to power sums of a negative degree of roots and their relation to residue integrals (multidimensional analogs of Waring's formulas) are obtained. Various examples of transcendental systems of equations and calculation of multidimensional numerical series are given.
\end{abstract}

Keywords: transcendental systems of equations, power sums of roots, residue integral.

Citation: A.M. Kytmanov, O.V. Khodos, On Transcendental Systems of Equations, J. Sib. Fed. Univ. Math. Phys., 2021, 14(3), 326-343. DOI: 10.17516/1997-1397-2021-14-3-326-343.

\section{Introduction}

Based on the multidimensional logarithmic residue, for systems of non-linear algebraic equations in $\mathbb{C}^{n}$ formulas for finding power sums of the roots of a system without calculating the roots themselves were earlier obtained (see [1-3]). For different types of systems such formulas have different forms. Based on this, a new method for the study of systems of algebraic equations in $\mathbb{C}^{n}$ have been constructed. It arose in the work of L. A. Aizenberg [1], its development was continued in monographs [2-4]. The main idea is to find power sums of roots of systems (for positive powers) and then, to use one-dimensional or multidimensional recurrent Newton formulas (see [5]). Unlike the classical method of elimination, it is less labor-intensive and does not increase the multiplicity of roots. It is based on the formula (see [1]) for a sum of the values of an arbitrary polynomial in the roots of a given systems of algebraic equations without finding the roots themselves.

For systems of transcendental equations, formulas for the sum of the values of the roots of the system, as a rule, cannot be obtained, since the number of roots of a system can be infinite and a series of coordinates of such roots can be diverging. Nevertheless, such transcendental systems of equations may very well arise, for example, in the problems of chemical kinetics [6,7]. Thus, this is an important task to consider such systems.

In the works [8-21] power sums of roots are considered for a negative power for different systems of non-algebraic (transcendental) equations. To compute these power sums, a residue integral is used, the integration is carried out over skeletons of polycircles centered at the origin. Note that this residue integral is not, generally speaking, a multidimensional logarithmic residue or a Grothendieck residue. For various types of lower homogeneous systems of functions included in the system, formulas are given for finding residue integrals, their relationship with power sums of the roots of the system to a negative degree are established.

\footnotetext{
*AKytmanov@sfu-kras.ru https://orcid.org/0000-0002-7394-1480

$\dagger$ khodos_o@mail.ru

(c) Siberian Federal University. All rights reserved
} 
The paper [12] investigated more complex systems in which the lower homogeneous parts are decomposed into linear factors and integration cycles in residue integrals are constructed from these factors. In [11], a system is studied that arises in the Zel'dovich-Semenov model (see [6,7]) in chemical kinetics.

The object of this study is transcendental systems of equations in which the lower homogeneous parts of the functions included in the system form a non-degenerate system of algebraic equations: formulas are found for calculating the residue integrals, power sums of roots for a negative power, their relationship with the residue integrals are established. See [21].

\section{The simplest transcendental systems of equations}

Consider a system of functions of the form

$$
f_{1}(z), f_{2}(z), \ldots, f_{n}(z)
$$

holomorphic in a neighborhood of the point $0 \in \mathbb{C}^{n}, z=\left(z_{1}, z_{2}, \ldots, z_{n}\right)$ and having the following form:

$$
f_{j}(z)=z^{\beta^{j}}+Q_{j}(z), \quad j=1,2, \ldots, n,
$$

where $\beta^{j}=\left(\beta_{1}^{j}, \beta_{2}^{j}, \ldots, \beta_{n}^{j}\right)$ is a multi-index with integer non-negative coordinates, $z^{\beta^{j}}=z_{1}^{\beta_{1}^{j}}$. $z_{2}^{\beta_{2}^{j}} \ldots z_{n}^{\beta_{n}^{j}}$ and $\left\|\beta^{j}\right\|=\beta_{1}^{j}+\beta_{2}^{j}+\ldots+\beta_{n}^{j}=k_{j}, j=1,2, \ldots, n$. The functions $Q_{j}$ can be expanded in absolutely and uniformly converging Taylor series in a neighborhood of the origin of the form

$$
Q_{j}(z)=\sum_{\|\alpha\|>k_{j}} a_{\alpha}^{j} z^{\alpha},
$$

where $\alpha=\left(\alpha_{1}, \alpha_{2}, \ldots, \alpha_{n}\right), \alpha_{j} \geqslant 0, \alpha_{j} \in \mathbb{Z}$, a $z^{\alpha}=z_{1}^{\alpha_{1}} \cdot z_{2}^{\alpha_{2}} \cdots z_{n}^{\alpha_{n}}$.

Consider the cycles $\gamma(r)=\gamma\left(r_{1}, r_{2}, \ldots, r_{n}\right)$, which are skeletons of polydisks:

$$
\gamma(r)=\left\{z \in \mathbb{C}^{n}:\left|z_{s}\right|=r_{s}, s=1,2, \ldots, n\right\}, \quad r_{1}>0, \ldots, r_{n}>0 .
$$

For sufficiently small $r_{j}$, the cycles $\gamma(r)$ lie in the domain of holomorphy of functions $f_{j}$, therefore the series

$$
\sum_{\|\alpha\|>k_{j}}\left|a_{\alpha}^{j}\right| r_{1}^{\alpha_{1}} \cdots r_{n}^{\alpha_{n}}, \quad j=1, \ldots n,
$$

converge. Then on the cycle $\gamma(t r)=\gamma\left(t r_{1}, t r_{2}, \ldots, t r_{n}\right)$ for sufficiently small $t>0$ we have

$$
|z|^{\beta^{j}}=t^{k_{j}} \cdot r_{1}^{\beta_{1}^{j}} \cdot r_{2}^{\beta_{2}^{j}} \cdots r_{n}^{\beta_{n}^{j}}=t^{k_{j}} \cdot r^{\beta^{j}},
$$

and

$$
\begin{gathered}
\left|Q_{j}(z)\right|=\left|\sum_{\|\alpha\|>k_{j}} a_{\alpha}^{j} z^{\alpha}\right| \leqslant \\
\leqslant \sum_{\|\alpha\|>k_{j}} t^{\|\alpha\|}\left|a_{\alpha}^{j}\right| r^{\alpha} \leqslant t^{k_{j}+1} \sum_{\|\alpha\| \geqslant 0}\left|a_{\alpha}^{j}\right| r^{\alpha}, \quad j=1, \ldots, n .
\end{gathered}
$$

Therefore, for such $t$ on the cycle $\gamma(t r)$ the inequalities hold

$$
|z|^{\beta^{j}}>\left|Q_{j}(z)\right|, \quad j=1,2, \ldots, n .
$$

Thus

$$
f_{j}(z) \neq 0 \quad \text { on } \quad \gamma(t r), \quad j=1,2, \ldots, n \text {. }
$$


In what follows, we will assume that $t=1$. Consider a system of equations of the form

$$
\left\{\begin{array}{l}
f_{1}(z)=0, \\
f_{2}(z)=0, \\
\cdots \cdots \cdots \\
f_{n}(z)=0 .
\end{array}\right.
$$

From (3) it follows that for sufficiently small $r_{j}$ the following integrals are defined

$$
\int_{\gamma(r)} \frac{1}{z^{\beta+I}} \cdot \frac{d f}{f}=\int_{\gamma\left(r_{1}, r_{2}, \ldots, r_{n}\right)} \frac{1}{z_{1}^{\beta_{1}+1} \cdot z_{2}^{\beta_{2}+1} \ldots z_{n}^{\beta_{n}+1}} \cdot \frac{d f_{1}}{f_{1}} \wedge \frac{d f_{2}}{f_{2}} \wedge \ldots \wedge \frac{d f_{n}}{f_{n}},
$$

where $\beta_{1} \geqslant 0, \beta_{2} \geqslant 0, \ldots, \beta_{n} \geqslant 0, \beta_{j} \in \mathbb{Z}, I=(1,1, \ldots, 1)$. We call them residue integrals ( [22]).

The logarithmic residue theorem does not apply to these integrals, and they are not standard Grothendieck residues.

Since condition (3) is satisfied on the cycles $\gamma(r)$, by the Cauchy-Poincaré theorem, these integrals are independent of $\left(r_{1}, \ldots, r_{n}\right)$. Let us denote

$$
J_{\beta}=\frac{1}{(2 \pi i)^{n}} \int_{\gamma(r)} \frac{1}{z^{\beta+I}} \cdot \frac{d f}{f} .
$$

Theorem 1. Under the assumptions made, for a function $f_{j}$ of the form (1), (2) the next formulas are valid

$$
\begin{gathered}
J_{\beta}=\sum_{\|\alpha\| \leqslant\|\beta\|+\min \left(n, k_{1}+\ldots+k_{n}\right)} \frac{(-1)^{\|\alpha\|}}{\left(\beta+\left(\alpha_{1}+1\right) \beta^{1}+\ldots+\left(\alpha_{n}+1\right) \beta^{n}\right) !} \times \\
\quad \times\left.\frac{\partial^{k}\left(\Delta \cdot Q^{\alpha}\right)}{\partial z^{\beta+\left(\alpha_{1}+1\right) \beta^{1}+\ldots+\left(\alpha_{n}+1\right) \beta^{n}}}\right|_{z=0}= \\
=\sum_{\|\alpha\| \leqslant\|\beta\|+\min \left(n, k_{1}+\ldots+k_{n}\right)}(-1)^{\|\alpha\|} \mathfrak{M}\left[\frac{\Delta \cdot Q^{\alpha}}{z^{\beta+\left(\alpha_{1}+1\right) \beta^{1}+\ldots+\left(\alpha_{n}+1\right) \beta^{n}}}\right],
\end{gathered}
$$

where $k=\left\|\beta+\left(\alpha_{1}+1\right) \beta^{1}+\ldots+\left(\alpha_{n}+1\right) \beta^{n}\right\|, \beta !=\beta_{1} ! \cdot \beta_{2} ! \cdots \beta_{n} !, Q^{\alpha}=Q_{1}^{\alpha_{1}} \cdot Q_{2}^{\alpha_{2}} \cdots Q_{n}^{\alpha_{n}}$, $\frac{\partial^{\|\beta\|}}{\partial z^{\beta}}=\frac{\partial^{\|\beta\|}}{\partial z_{1}^{\beta_{1}} \partial z_{2}^{\beta_{2}} \cdots \partial z_{n}^{\beta_{n}}}, \Delta$ is the Jacobian of the system of functions (1) and, finally, $\mathfrak{M}$ is a linear functional assigning to the Laurent series (under the sign of the functional $\mathfrak{M}$ ) its free term.

Corollary 1. If all $\beta^{j}=(0,0, \ldots, 0), j=1, \ldots, n$, then the integral

$$
J_{\beta}=\sum_{\|\alpha\| \leqslant\|\beta\|}(-1)^{\|\alpha\|} \mathfrak{M}\left[\frac{\Delta \cdot Q^{\alpha}}{z^{\beta}}\right]=\left.\sum_{\|\alpha\| \leqslant\|\beta\|} \frac{(-1)^{\|\alpha\|}}{\beta !} \frac{\partial^{\|\beta\|}}{\partial z^{\beta}}\left(\Delta \cdot Q^{\alpha}\right)\right|_{z=0} .
$$

Our further goal is to relate the considered integrals to power sums of roots of the system (4). To do this, we will narrow the function class $f_{j}$. First, we take as functions $Q_{j}(j=1,2, \ldots, n)$ polynomials of the form

$$
Q_{j}(z)=\sum_{\alpha \in M_{j}} a_{\alpha}^{j} z^{\alpha}
$$


where $M_{j}$ is a finite set of multi-indices such that for $\alpha \in M_{j}$ the coordinates $\alpha_{k} \leqslant \beta_{k}^{j}$, $k=1,2, \ldots, n, k \neq j$. (But it is still assumed that $\|\alpha\|>k_{j}$ for all $\alpha \in M_{j}$ ).

Denote

$$
\sigma_{\beta+I}=\sigma_{\left(\beta_{1}+1, \beta_{2}+1, \ldots, \beta_{n}+1\right)}=\sum_{k=1}^{M} \frac{1}{z_{1(k)}^{\beta_{1}+1} \cdot z_{2(k)}^{\beta_{2}+1} \cdots z_{n(k)}^{\beta_{n}+1}},
$$

where $\beta=\left(\beta_{1}, \ldots, \beta_{n}\right)$ is some multi-index. This expression is a power sum of roots that do not lie on the coordinate planes of the system (4), but in negative power (or a power sum of the reciprocal of the roots).

Theorem 2. For the system (4) with functions $f_{j}$ of the form (1) and polynomials $Q_{j}$ of the form (5) the next formulas are valid

$$
J_{\beta}=(-1)^{n} \sigma_{\beta+I},
$$

i.e.

$$
\sigma_{\beta+I}=\sum_{\|\alpha\| \leqslant\|\beta\|+\min \left(n, k_{1}+\ldots+k_{n}\right)}(-1)^{\|\alpha\|+n} \mathfrak{M}\left[\frac{\Delta \cdot Q^{\alpha}}{z^{\beta+\left(\alpha_{1}+1\right) \beta^{1}+\ldots+\left(\alpha_{n}+1\right) \beta^{n}}}\right] .
$$

Consider a system of equations in three complex variables

$$
\left\{\begin{array}{l}
f_{1}\left(z_{1}, z_{2}, z_{3}\right)=1+a_{1} z_{1}=0 \\
f_{2}\left(z_{1}, z_{2}, z_{3}\right)=1+b_{1} z_{1}+b_{2} z_{2}=0 \\
f_{3}\left(z_{1}, z_{2}, z_{3}\right)=1+c_{1} z_{1}+c_{2} z_{2}+c_{3} z_{3}=0 .
\end{array}\right.
$$

Here the functions do not satisfy the conditions of Theorem 2, but they satisfy the conditions of Theorem 1. We find the integral

$$
\begin{gathered}
J_{(\beta, 0,0)}=\frac{1}{(2 \pi i)^{3}} \int_{\gamma(r)} \frac{1}{z_{1}^{\beta_{1}+1} z_{2} z_{3}} \cdot \frac{d f_{1} \wedge d f_{2} \wedge d f_{3}}{f_{1} \cdot f_{2} \cdot f_{3}}= \\
=\frac{1}{(2 \pi i)^{3}} \int_{\gamma(r)} \frac{1}{z_{1}^{\beta_{1}+1} z_{2} z_{3}} \cdot \frac{a_{1} b_{2} c_{3} d z_{1} \wedge d z_{2} \wedge d z_{3}}{\left(1+a_{1} z_{1}\right)\left(1+b_{1} z_{1}+b_{2} z_{2}\right)\left(1+c_{1} z_{1}+c_{2} z_{2}+c_{3} z_{3}\right)}= \\
=\left.\frac{a_{1} b_{2} c_{3}}{\beta !} \cdot \frac{\partial^{\beta}}{\partial z_{1}^{\beta}} \cdot\left[\frac{1}{\left(1+a_{1} z_{1}\right)\left(1+b_{1} z_{1}\right)\left(1+c_{1} z_{1}\right)}\right]\right|_{z_{1}=0} .
\end{gathered}
$$

To calculate the last derivative, we transform the expression

$$
\begin{gathered}
\frac{1}{\left(1+a_{1} z_{1}\right)\left(1+b_{1} z_{1}\right)\left(1+c_{1} z_{1}\right)}=\frac{A}{1+a_{1} z_{1}}+\frac{B}{1+b_{1} z_{1}}+\frac{C}{1+c_{1} z_{1}}, \\
\left\{\begin{array}{l}
A=\frac{a_{1}^{2}}{\left(a_{1}-b_{1}\right)\left(a_{1}-c_{1}\right)}, \\
B=-\frac{b_{1}^{2}}{\left(a_{1}-b_{1}\right)\left(b_{1}-c_{1}\right)}, \\
C=\frac{c_{1}^{2}}{\left(a_{1}-c_{1}\right)\left(b_{1}-c_{1}\right)},
\end{array}\right.
\end{gathered}
$$

assuming that $a_{1} \neq b_{1}, a_{1} \neq c_{1}, b_{1} \neq c_{1}$, then

$$
J_{(\beta, 0,0)}=(-1)^{\beta} a_{1} b_{2} c_{3} \times
$$




$$
\times\left[\frac{a_{1}^{\beta+2}}{\left(a_{1}-b_{1}\right)\left(a_{1}-c_{1}\right)}-\frac{b_{1}^{\beta+2}}{\left(a_{1}-b_{1}\right)\left(b_{1}-c_{1}\right)}+\frac{c_{1}^{\beta+2}}{\left(a_{1}-c_{1}\right)\left(b_{1}-c_{1}\right)}\right] .
$$

The roots of the system (6)

$$
z_{1}=-\frac{1}{a_{1}}, \quad z_{2}=\frac{b_{1}-a_{1}}{a_{1} b_{2}}, \quad z_{3}=\frac{b_{2} c_{1}-b_{1} c_{2}+a_{1} c_{2}-a_{1} b_{2}}{a_{1} b_{2} c_{3}} .
$$

If the numerator in the formula for $z_{3}$ is 0 , then this root lies on a coordinate plane, and we should not take it into consideration.

Therefore, the power sum

$$
\sigma_{\left(\beta_{1}+1,1\right)}=\frac{(-1)^{\beta+1} a_{1}^{\beta+3} b_{2}^{2} c_{3}}{\left(b_{1}-a_{1}\right)\left(b_{2} c_{1}-b_{1} c_{2}+a_{1} c_{2}-a_{1} b_{2}\right)},
$$

i.e.

$$
\begin{gathered}
J_{(\beta, 0,0)}=-\sigma_{\left(\beta_{1}+1,1\right)}-\frac{(-1)^{\beta} a_{1}^{2} b_{2}^{2} c_{3} b_{1}^{\beta+1}}{\left(b_{1}-a_{1}\right)\left(b_{2} c_{1}-b_{1} c_{2}+a_{1} c_{2}-a_{1} b_{2}\right)}+ \\
+\frac{(-1)^{\beta+1} a_{1} b_{2} c_{2} c_{3}}{\left(b_{2} c_{1}-b_{1} c_{2}+a_{1} c_{2}-a_{1} b_{2}\right)} \times \\
\times\left[-a_{1} c_{2} \cdot \frac{a_{1}^{\beta+1}-c_{1}^{\beta+1}}{a_{1}-c_{1}}+\left(b_{1} c_{2}-b_{2} c_{1}\right) \cdot \frac{b_{1}^{\beta+1}-c_{1}^{\beta+1}}{b_{1}-c_{1}}\right] .
\end{gathered}
$$

We recall the well-known expansions of the sine into an infinite product and the power series:

$$
\frac{\sin \sqrt{z}}{\sqrt{z}}=\prod_{k=1}^{\infty}\left(1-\frac{z}{k^{2} \pi^{2}}\right)=\sum_{k=0}^{\infty} \frac{(-1)^{k} z^{k}}{(2 k+1) !}
$$

which converge uniformly and absolutely on the complex plane.

Consider the system of equations

$$
\left\{\begin{array}{l}
f_{1}\left(z_{1}, z_{2}, z_{3}\right)=\frac{\sin \sqrt{z_{1}-a^{2}}}{\sqrt{z_{1}-a^{2}}}=\prod_{k=1}^{\infty}\left(1-\frac{z_{1}-a^{2}}{k^{2} \pi^{2}}\right)=0 \\
f_{2}\left(z_{1}, z_{2}, z_{3}\right)=\frac{\sin \sqrt{z_{2}-z_{1}-a^{2}}}{\sqrt{z_{2}-z_{1}-a^{2}}}=\prod_{m=1}^{\infty}\left(1-\frac{z_{2}-z_{1}-a^{2}}{m^{2} \pi^{2}}\right)=0 \\
f_{3}\left(z_{1}, z_{2}, z_{3}\right)=\frac{\sin \sqrt{z_{3}-z_{2}-a^{2}}}{\sqrt{z_{3}-z_{2}-a^{2}}}=\prod_{s=1}^{\infty}\left(1-\frac{z_{3}-z_{2}-a^{2}}{s^{2} \pi^{2}}\right)=0 .
\end{array}\right.
$$

Each of the functions of this system can be expanded into an infinite product of functions from system (6).

The roots of the system (9) are the points $\left(\pi^{2} k^{2}+a^{2}, \pi^{2}\left(k^{2}+m^{2}\right)+2 a^{2}, \pi^{2}\left(k^{2}+m^{2}+s^{2}\right)+3 a^{2}\right)$, $k, m, s \in \mathbb{N}$. Therefore, the power sum $\sigma_{(\beta+1,1,1)}$ is equal to the sum of the series

$$
\sigma_{(\beta+1,1,1)}=\sum_{k, m, s=1}^{\infty} \frac{1}{\left(\pi^{2} k^{2}+a^{2}\right)^{(\beta+1)}\left(\pi^{2}\left(k^{2}+m^{2}\right)+2 a^{2}\right)\left(\pi^{2}\left(k^{2}+m^{2}+s^{2}\right)+3 a^{2}\right)},
$$

which converges as $a \neq \pi k i$.

For the system (9)

$$
f_{1}=\sum_{k=0}^{\infty} \frac{(-1)^{k}\left(z_{1}-a^{2}\right)^{k}}{(2 k+1) !}
$$




$$
\begin{aligned}
& f_{2}=\sum_{k=0}^{\infty} \frac{(-1)^{k}\left(z_{2}-z_{1}-a^{2}\right)^{k}}{(2 k+1) !}, \\
& f_{3}=\sum_{k=0}^{\infty} \frac{(-1)^{k}\left(z_{3}-z_{2}-a^{2}\right)^{k}}{(2 k+1) !},
\end{aligned}
$$

therefore

$$
f_{1}(0,0,0)=f_{2}(0,0,0)=f_{3}(0,0,0)=\sum_{k=0}^{\infty} \frac{a^{2} k}{(2 k+1) !}=\frac{\text { sha }}{a} .
$$

Therefore, to apply the formula from Theorem 1, we need to divide the functions $f_{1}, f_{2}, f_{3}$ by these constants (normalize).

Consider the integral $J_{(\eta, 0,0)}$ for the system (9). Using the form of the roots of the system (9), we obtain that

$$
\begin{gathered}
a_{1}=-\frac{1}{\pi^{2} k^{2}+a^{2}}, \quad b_{1}=\frac{1}{\pi^{2} m^{2}+a^{2}}, \quad b_{2}=-\frac{1}{\pi^{2} m^{2}+a^{2}}, \quad c_{2}=\frac{1}{\pi^{2} s^{2}+a^{2}}, \quad c_{3}=-\frac{1}{\pi^{2} s^{2}+a^{2}} . \\
\quad \times \sum_{(\beta, 0,0)}^{\infty} \frac{\sigma_{(\beta+1,1,1)}+(-1)^{\beta+1} \times}{\left(m^{2} \pi^{2}+a^{2}\right)^{\beta+1}\left(\pi^{2}\left(k^{2}+m^{2}\right)+2 a^{2}\right)\left(\pi^{2}\left(k^{2}+m^{2}+s^{2}\right)+3 a^{2}\right)}+ \\
+(-1)^{\beta+1} \sum_{k, m, s=1}^{\infty} \frac{1}{\left(\pi^{2} s^{2}+a^{2}\right)\left(\pi^{2}\left(k^{2}+m^{2}+s^{2}\right)+3 a^{2}\right)} \times\left[\frac{1}{\left(m^{2} \pi^{2}+a^{2}\right)^{\beta+1}}+\frac{(-1)^{\beta}}{\left(k^{2} \pi^{2}+a^{2}\right)^{\beta+1}}\right], \\
J_{(\beta, 0,0)}=\sum_{k, m, s=1}^{\infty} \frac{1}{\left(\pi^{2}\left(k^{2}+m^{2}\right)+2 a^{2}\right)\left(\pi^{2}\left(k^{2}+m^{2}+s^{2}\right)+3 a^{2}\right)} \times \\
\left.\times(-1)^{\beta+1} \sum_{k, m, s=1}^{\infty} \frac{-1}{\left(k^{2} \pi^{2}+a^{2}\right)^{\beta+1}}+\frac{(-1)^{\beta+1}}{\left(m^{2} \pi^{2}+a^{2}\right)^{\beta+1}}\right]+ \\
\times \quad \times\left[\frac{1}{\left(m^{2} \pi^{2}+a^{2}\right)^{\beta+1}}+\frac{(-1)^{\beta}}{\left(k^{2} \pi^{2}+a^{2}\right)^{\beta+1}}\right] .
\end{gathered}
$$

For odd $\beta$ the integral $J_{(\beta, 0,0)}=0$, and for even $\beta=2 n$ we obtain the following formula for finding the sum of the series

$$
\begin{gathered}
J_{(2 n, 0,0)}=\sum_{\|\alpha\| \leqslant 2 n} \mathfrak{M}\left[\frac{\Delta \cdot Q^{\alpha}}{z_{1}^{2 n}}\right]=-2 \sigma_{(2 n+1,1,1)}- \\
-2 \sum_{k, m, s=1}^{\infty} \frac{1}{\left(\pi^{2} k^{2}+a^{2}\right)^{(2 n+1)} \cdot\left(\pi^{2} s^{2}+a^{2}\right) \cdot\left(\pi^{2}\left(k^{2}+m^{2}+s^{2}\right)+3 a^{2}\right)} .
\end{gathered}
$$

Let us calculate, for example:

$$
J_{(0,0,0)}=\mathfrak{M}[\Delta]=\mathfrak{M}\left[\frac{\partial f_{1}}{\partial z_{1}} \cdot \frac{\partial f_{2}}{\partial z_{2}} \cdot \frac{\partial f_{3}}{\partial z_{3}}\right]=\left(\frac{1}{2 a^{2}}-\frac{1}{2 a} \text { ctha }\right)^{3} .
$$

Applying the identity

$$
\frac{1}{\left(\pi^{2} k^{2}+a^{2}\right)\left(\pi^{2}\left(k^{2}+m^{2}\right)+2 a^{2}\right)}+\frac{1}{\left(\pi^{2} m^{2}+a^{2}\right)\left(\pi^{2}\left(k^{2}+m^{2}\right)+2 a^{2}\right)}=
$$




$$
=\frac{1}{\left(\pi^{2} k^{2}+a^{2}\right)\left(\pi^{2} m^{2}+a^{2}\right)}
$$

We get that

$$
2 \sigma_{(1,1,1)}=\sum_{k, m, s=1}^{\infty} \frac{1}{\left(\pi^{2} k^{2}+a^{2}\right) \cdot\left(\pi^{2} s^{2}+a^{2}\right) \cdot\left(\pi^{2}\left(k^{2}+m^{2}+s^{2}\right)+3 a^{2}\right)} .
$$

Thus, we get

$$
\begin{gathered}
\left.\sum_{k, m, s=1}^{\infty} \frac{1}{\left(\pi^{2} k^{2}+a^{2}\right) \cdot\left(\pi^{2}\left(k^{2}+m^{2}\right)+2 a^{2}\right) \cdot\left(\pi^{2}\left(k^{2}+m^{2}+s^{2}\right)+3 a^{2}\right)}\right)= \\
=\frac{(a \text { ctha }-1)^{3}}{48 a^{6}} .
\end{gathered}
$$

\section{Special systems of equations}

Consider a system of functions $f_{1}(z), f_{2}(z), \ldots, f_{n}(z)$ of the form

$$
\left\{\begin{array}{l}
f_{1}(z)=\left(1-a_{11} z_{1}\right)^{m_{11}} \cdots\left(1-a_{1 n} z_{n}\right)^{m_{1 n}}+Q_{1}(z) \\
f_{2}(z)=\left(1-a_{21} z_{1}\right)^{m_{21}} \cdots\left(1-a_{2 n} z_{n}\right)^{m_{2 n}}+Q_{2}(z) \\
\cdots \\
f_{n}(z)=\left(1-a_{n 1} z_{1}\right)^{m_{n 1}} \cdots\left(1-a_{n n} z_{n}\right)^{m_{n n}}+Q_{n}(z)
\end{array}\right.
$$

where $m_{i j}$ are natural numbers, $a_{i j}$ are complex numbers that are different for fixed $j, Q_{i}(z)$ are entire functions, $i=1, \ldots, n$. Let $J=\left(j_{1}, \ldots, j_{n}\right)$ be a multi-index, where $\left(j_{1} \ldots j_{n}\right)$ is a permutation of $(1, \ldots, n)$. Let us define $a_{J}=\left(a_{1 j_{1}}, \ldots, a_{n j_{n}}\right)$ for a multi-index $J$. We denote

$$
q_{i}\left(z_{1}, \ldots, z_{n}\right)=\left(1-a_{i 1} z_{1}\right)^{m_{i 1}} \ldots\left(1-a_{i n} z_{n}\right)^{m_{i n}}, \quad i=1, \ldots, n,
$$

then the system (10) can be rewritten as

$$
f_{i}\left(z_{1}, \ldots, z_{n}\right)=q_{i}\left(z_{1}, \ldots, z_{n}\right)+Q_{i}\left(z_{1}, \ldots, z_{n}\right), \quad i=1, \ldots, n
$$

For each $m$ we define the function

$$
h_{m}(z)= \begin{cases}q_{m}(z) & \text { if } a_{m j} \neq 0 \text { for all } j \\ q_{m}(z) \cdot \frac{1}{z_{j_{1}}} \cdot \ldots \cdot \frac{1}{z_{j_{k}}} & \text { if } a_{m j_{1}}=\ldots=a_{i j_{k}}=0\end{cases}
$$

A system

$$
h_{m}(z)=0, \quad i=1, \ldots, n,
$$

has $n$ ! isolated roots in $\overline{\mathbb{C}}^{n}$, where $\overline{\mathbb{C}}^{n}=\overline{\mathbb{C}} \times \cdots \times \overline{\mathbb{C}}$. Recall that $\overline{\mathbb{C}}$ is a compactification of the complex plane $\mathbb{C}$ (the Riemann sphere). Then $\overline{\mathbb{C}}^{n}$ is one of the well-known compactifications of $\mathbb{C}^{n}$ (the function theory space). The roots of the system (14) are equal

$$
\tilde{a}_{J}= \begin{cases}\left(1 / a_{1 j_{1}}, \ldots, 1 / a_{n j_{n}}\right) & \text { if } a_{k j_{k}} \neq 0 \text { for } k=1, \ldots, n \\ \left(1 / a_{1 j_{1}}, \ldots, \infty_{\left[i_{1}\right]}, \ldots, \infty_{\left[i_{k}\right]}, \ldots, 1 / a_{n j_{n}}\right) & \text { if } a_{i_{1} j_{i_{1}}}=\ldots=a_{i_{k} j_{i_{k}}}=0\end{cases}
$$

where $k, j=1, \ldots, n$. If $a_{j_{1}, i_{1}}=0$, then in $\tilde{a}_{J}$ we write $\infty$, this is the point at infinity in $\overline{\mathbb{C}}$. 
By $\Gamma_{h}$ we denote the (global) cycle:

$$
\Gamma_{h}=\left\{z \in \mathbb{C}^{n}:\left|h_{m}\right|=r_{m}, r_{i}>0, m=1, \ldots, n\right\} .
$$

In the case when all $a_{k, j_{k}} \neq 0$, we define the (local) cycle $\Gamma_{h, \tilde{a}_{J}}$ as follows

$$
\left\{\begin{array}{l}
\left|1-a_{1 j_{1}} z_{1}\right|=r_{1}, \\
\left|1-a_{2 j_{2}} z_{2}\right|=r_{2}, \\
\cdots \\
\left|1-a_{n j_{n}} z_{n}\right|=r_{n} .
\end{array}\right.
$$

If $a_{i_{1} j_{i_{1}}}=\ldots=a_{i_{k} j_{i_{k}}}=0$ for some $i_{1}, \ldots, i_{k}$, then $\Gamma_{h, \tilde{a}_{J}}$ is defined as

$$
\left\{\begin{array}{l}
\left|1-a_{1 j_{1}} z_{1}\right|=r_{1}, \\
\cdots \\
\left|1 / z_{i_{1}}\right|=r_{i_{1}}, \\
\cdots \\
\left|1 / z_{i_{k}}\right|=r_{i_{k}}, \\
\cdots \\
\left|1-a_{n j_{n}} z_{n}\right|=r_{n} .
\end{array}\right.
$$

Lemma 1. For sufficiently small $r_{m}$, the global cycle $\Gamma_{h}$ has connected components (local cycles) in the neighborhood of the roots $a_{J}$. Moreover, $\Gamma_{h}$ is homologous to the sum of local cycles $\Gamma_{h, \tilde{a}_{J}}$.

Consider the system of equations

$$
F_{m}(z, t)=q_{m}(z)+t \cdot Q_{m}(z)=0, \quad m=1, \ldots, n,
$$

depending on the real parameter $t \geqslant 0$. Let $r_{1}>0, \ldots, r_{n}>0$ be fixed real numbers. Then, for sufficiently small $t>0$, the inequalities

$$
\left|q_{m}(z)\right|>\left|t \cdot Q_{m}(z)\right|, \quad m=1, \ldots, n
$$

on cycles

$$
\Gamma_{h}=\left\{z \in \mathbb{C}^{n}:\left|h_{m}\right|=r_{m}, \quad m=1, \ldots, n\right\}
$$

because $\Gamma_{h}$ is compact.

We denote by $J_{\gamma}(t)$ the residue integral

$$
\begin{gathered}
J_{\gamma}(t)=\frac{1}{(2 \pi i)^{n}} \int_{\Gamma_{h}} \frac{1}{z^{\gamma+I}} \cdot \frac{d F}{F}= \\
=\frac{1}{(2 \pi i)^{n}} \int_{\Gamma_{h}} \frac{1}{z_{1}^{\gamma_{1}+1} \cdot z_{2}^{\gamma_{2}+1} \cdots z_{n}^{\gamma_{n}+1}} \cdot \frac{d F_{1}}{F_{1}} \wedge \frac{d F_{2}}{F_{2}} \wedge \ldots \wedge \frac{d F_{n}}{F_{n}},
\end{gathered}
$$

where $\gamma=\left(\gamma_{1}, \ldots, \gamma_{n}\right)$ is a multi-index, and $I=(1,1, \ldots, 1)$.

We denote by $\Delta=\Delta(t)$ the Jacobian of the system of functions $F_{1}(z, t), \ldots, F_{n}(z, t)$ in the variables $z_{1}, \ldots, z_{n}$. 
Theorem 3. Under the assumptions made on the functions $F_{i}$ defined by formulas (18), the following expressions for $J_{\gamma}(t)$ are absolutely convergent (for sufficiently small $t$ ) series:

$$
\begin{gathered}
J_{\gamma}(t)=\sum_{J}^{\prime} \sum_{\alpha} \frac{(-1)^{s(J)}(-t)^{\|\alpha\|+\| \beta(\alpha, J) \mid+n}}{\beta(\alpha, J) ! \cdot a_{J}^{\beta+I}} \times \\
\times\left.\frac{\partial^{\| \beta(\alpha(J) \|}}{\partial z^{\beta(\alpha, J)}}\left[\frac{\Delta(t)}{z_{1}^{\gamma_{1}+1} \cdot \ldots \cdot z_{n}^{\gamma_{n}+1}} \cdot \frac{Q^{\alpha}}{q^{\alpha+I}(J)}\right]\right|_{z=\tilde{a}_{J}},
\end{gathered}
$$

where $(-1)^{s(J)}$ is the parity of the permutation $J, \alpha=\left(\alpha_{1}, \ldots, \alpha_{n}\right)$ is a multi-index of length $n$, $q^{\alpha+I}(J)=q_{1}^{\alpha_{1}+1}\left[j_{1}\right] \cdots q_{n}^{\alpha_{n}+1}\left[j_{n}\right]$, and $q_{s}\left[j_{s}\right]$ is the product of all $\left(1-a_{j 1} z_{1}\right)^{m_{j 1}} \cdots\left(1-a_{j n} z_{n}\right)^{m_{j n}}$, except $\left(1-a_{s j_{s}} z_{s}\right)^{m_{s j_{s}}}$,

$$
\begin{gathered}
\beta(\alpha, J)=\left(m_{1 j_{1}}\left(\alpha_{j_{1}}+1\right)-1, \ldots, m_{n j_{n}}\left(\alpha_{j_{n}}+1\right)-1\right), \\
\beta(\alpha, J) !=\prod_{p}\left(m_{p j_{p}}\left(\alpha_{j_{p}}+1\right)-1\right) !, \\
a_{j}^{\beta+I}=a_{1 j_{1}}^{m_{1 j_{1}}\left(\alpha_{j_{1}}+1\right)} \cdots a_{n j_{n}}^{m_{n j_{n}}\left(\alpha_{j_{n}}+1\right)}, \\
\frac{\partial^{\| \beta(\alpha(J) \|}}{\partial z^{\beta(\alpha, J)}}=\frac{\partial^{m_{1 j_{1}}\left(\alpha_{j_{1}}+1\right)-1+\ldots+m_{n j_{n}}\left(\alpha_{j_{n}}+1\right)-1}}{\partial z_{1}^{m_{1 j_{1}}\left(\alpha_{j_{1}}+1\right)-1} \ldots \partial z_{n}^{m_{n j_{n}}\left(\alpha_{j_{n}}+1\right)-1} .}
\end{gathered}
$$

The dash at the summation sign means that the summation is performed over all multi-indices $J$ for which there are no zero coordinates in $a_{J}$.

Suppose $Q_{s}(z)$ are polynomials:

$$
Q_{s}(z)=z_{1} \cdots z_{n} \sum_{\mid \alpha \| \geqslant 0} C_{\alpha}^{s} z^{\alpha} \quad s=1, \ldots, n,
$$

where $\alpha$ is a multi-index, $z^{\alpha}=z_{1}^{\alpha_{1}} \cdots z_{n}^{\alpha_{n}}$, and $\operatorname{deg}_{z_{j}} Q_{s} \leqslant m_{s j}, s, j=1, \ldots, n$ for all nonzero $a_{s j}$. If $a_{s j}=0$, then there are no restrictions on the degree $\operatorname{deg}_{z_{j}} Q_{s}$.

Assuming that all $w_{j} \neq 0$, we make the change $z_{j}=\frac{1}{w_{j}}, j=1, \ldots, n$ in the functions

$$
F_{s}(z, t)=\left(q_{s}(z)+t \cdot Q_{s}(z)\right), \quad s=1, \ldots, n .
$$

Hence, for $s=1, \ldots, n$ we have

$$
\begin{gathered}
F_{s}\left(\frac{1}{w_{1}}, \ldots, \frac{1}{w_{n}}, t\right)=q_{s}\left(\frac{1}{w_{1}}, \ldots, \frac{1}{w_{n}}\right)+t \cdot Q_{s}\left(\frac{1}{w_{1}}, \ldots, \frac{1}{w_{n}}\right)= \\
=\left(1-a_{s 1} \frac{1}{w_{1}}\right)^{m_{s 1}} \cdots\left(1-a_{s n} \frac{1}{w_{n}}\right)^{m_{s n}}+t \cdot Q_{s}\left(\frac{1}{w_{1}}, \ldots, \frac{1}{w_{n}}\right)= \\
=\left(\frac{1}{w_{1}}\right)^{m_{s 1}} \cdots\left(\frac{1}{w_{n}}\right)^{m_{s n}} \cdot\left(w_{1}-a_{s 1}\right)^{m_{s 1}} \cdots\left(w_{n}-a_{s n}\right)^{m_{s n}}+t \cdot Q_{s}\left(\frac{1}{w_{1}}, \ldots, \frac{1}{w_{n}}\right) .
\end{gathered}
$$

Then we arrive at the formula

$$
F_{s}\left(\frac{1}{w_{1}}, \ldots, \frac{1}{w_{n}}, t\right)=\left(\frac{1}{w_{1}}\right)^{m_{s 1}} \cdots\left(\frac{1}{w_{n}}\right)^{m_{s n}} \cdot\left(\widetilde{q}_{s}(w)+t \cdot \widetilde{Q}_{s}(w)\right),
$$

where $\widetilde{q}_{s}$ are functions

$$
\widetilde{q}_{s}=\left(w_{1}-a_{s 1}\right)^{m_{s 1}} \cdots\left(w_{n}-a_{s n}\right)^{m_{s n}},
$$


and $\widetilde{Q}_{i}$ are polynomials

$$
\widetilde{Q}_{s}=w_{1}^{m_{s 1}} \cdots w_{n}^{m_{s n}} \cdot Q_{s}\left(\frac{1}{w_{1}}, \ldots, \frac{1}{w_{n}}\right) .
$$

From the formula (20) we obtain

$$
\operatorname{deg}_{w_{j}} \widetilde{Q}_{s}<m_{s j}, \quad s, j=1, \ldots, n .
$$

We denote

$$
\widetilde{F_{s}}=\widetilde{F_{s}}(w, t)=\widetilde{q}_{s}(w)+t \cdot \widetilde{Q}_{s}(w), \quad s=1, \ldots, n .
$$

If $0 \leqslant t \leqslant 1$, then the system (22) has a finite number of roots in $\mathbb{C}^{n}$ that depend on $t$. Moreover, (22) has no infinite roots in $\overline{\mathbb{C}}^{n}$.

Consider the cycle

$$
\widetilde{\Gamma}_{h}=\left\{w \in \mathbb{C}^{n}:\left|h_{s}\left(\frac{1}{w_{1}}, \ldots, \frac{1}{w_{n}}\right)\right|=\varepsilon_{s}, \quad s=1, \ldots, n\right\},
$$

for $t$ close enough to zero. The compactness of the cycle $\widetilde{\Gamma}_{h}$ implies

$$
\left|q_{s}\left(\frac{1}{w_{1}}, \ldots, \frac{1}{w_{n}}\right)\right|>\left|t \cdot Q_{s}\left(\frac{1}{w_{1}}, \ldots, \frac{1}{w_{n}}\right)\right|, \quad s=1, \ldots, n .
$$

Therefore, $\widetilde{\Gamma}_{h}$ is homologous to the sum of cycles $\widetilde{\Gamma}_{h, \tilde{a}_{J}}$ :

$$
\left\{\begin{array}{l}
\left|1-a_{1 j_{1}} \frac{1}{w_{1}}\right|=\varepsilon_{1}, \\
\left|1-a_{2 j_{2}} \frac{1}{w_{2}}\right|=\varepsilon_{2}, \\
\ldots \ldots \ldots \ldots \\
\left|1-a_{n j_{n}} \frac{1}{w_{n}}\right|=\varepsilon_{n},
\end{array}\right.
$$

obtained from the cycles $\Gamma_{h, \tilde{a}_{J}}$ by replacing $z_{j}=\frac{1}{w_{j}}$.

The equation

defines a circle. Indeed, we rewrite it as

$$
\left|1-a_{j s_{j}} \frac{1}{w_{j}}\right|=\varepsilon
$$

$$
\left|w_{j}-a_{j s_{j}}\right|=\varepsilon\left|w_{j}\right| \quad \text { or } \quad\left|w_{j}-a_{j s_{j}}\right|^{2}=\varepsilon^{2}\left|w_{j}\right|^{2},
$$

then

or

$$
\left(1-\varepsilon^{2}\right)\left|w_{j}-\frac{a_{j s_{j}}}{1-\varepsilon^{2}}\right|^{2}=\frac{\varepsilon^{2} \cdot\left|a_{j s_{j}}\right|^{2}}{\left(1-\varepsilon^{2}\right)}
$$

$$
\left|w_{j}-\frac{a_{j s_{j}}}{1-\varepsilon^{2}}\right|^{2}=\frac{\varepsilon^{2} \cdot\left|a_{j s_{j}}\right|^{2}}{\left(1-\varepsilon^{2}\right)^{2}}, \quad j=1, \ldots, n,
$$

for sufficiently small $\varepsilon$ the point $a_{j s_{j}}$ lies outside the circle and, therefore, $\widetilde{\Gamma}_{h, \tilde{a}_{J}}$ is homologous to the cycle $\widetilde{\Gamma}_{h, a_{J}}$ :

Here some $a_{i j}$ can be zero.

$$
\left\{\begin{array}{l}
\left|w_{1}-a_{1 j_{1}}\right|=\varepsilon_{1} \\
\left|w_{2}-a_{2 j_{2}}\right|=\varepsilon_{2} \\
\ldots \ldots \ldots . \\
\left|w_{n}-a_{n j_{n}}\right|=\varepsilon_{n}
\end{array}\right.
$$


Lemma 2. The residue integral (19) is

$$
J_{\gamma}(t)=\frac{(-1)^{n}}{(2 \pi i)^{n}} \int_{\widetilde{\Gamma}_{h}} w_{1}^{\gamma_{1}+1} \cdot w_{2}^{\gamma_{2}+1} \cdots w_{n}^{\gamma_{n}+1} \cdot \frac{d \widetilde{F_{1}}}{\widetilde{F_{1}}} \wedge \frac{d \widetilde{F_{2}}}{\widetilde{F_{2}}} \wedge \ldots \wedge \frac{d \widetilde{F}_{n}}{\widetilde{F_{n}}} .
$$

Theorem 4. The following equalities are valid

$$
\begin{gathered}
\sum_{j=1}^{p} \frac{1}{z_{j 1}(t)^{\gamma_{1}+1} \cdot z_{j 2}(t)^{\gamma_{2}+1} \cdots z_{j n}(t)^{\gamma_{n}+1}}= \\
=\sum_{K \in \Re}(-t)^{\|K\|+n} \sum_{J} \frac{(-1)^{s(J)}}{\beta(K, J) !} \cdot \frac{\partial^{\|\beta(K, J)\|}}{\partial w^{\beta}(K, J)} \times\left.\left[\widetilde{\Delta}(t) \cdot w_{1}^{\gamma_{1}+1} \cdots w_{n}^{\gamma_{n}+1} \cdot \frac{\widetilde{Q}^{K}}{\widetilde{q}^{K+I}(J)}\right]\right|_{w=a_{J}} .
\end{gathered}
$$

Since zeros of (22) are polynomials in $t$, the equality (4) also holds for $t=1$. We denote

$$
\sigma_{\gamma+I}=\sum_{j=1}^{p} \frac{1}{z_{j 1}^{\gamma_{1}+1} \cdot z_{j 2}^{\gamma_{2}+1} \cdots z_{j n}^{\gamma_{n}+1}}
$$

where $z^{(j)}=\left(z_{j 1}, \ldots, z_{j n}\right)=\left(z_{j 1}(1), \ldots, z_{j n}(1)\right), j=1, \ldots, n$.

Theorem 5. For the system (10) with functions $f_{j}$ defined in (12) and $Q_{i}$ defined in (20), the following formulas hold:

$$
\begin{gathered}
\sigma_{\gamma+I}=\sum_{j=1}^{p} \frac{1}{z_{j 1}^{\gamma_{1}+1} \cdot z_{j 2}^{\gamma_{2}+1} \cdots z_{j n}^{\gamma_{n}+1}}= \\
=\frac{1}{(2 \pi i)^{n}} \sum_{\|K\| \geqslant 0}(-1)^{\|K\|+n} \sum_{J}(-1)^{s(J)} \times \\
\times \int_{\widetilde{\Gamma}_{h, a}} \widetilde{\Delta} \cdot w_{1}^{\gamma_{1}+1} \ldots w_{n}^{\gamma_{n}+1} \cdot \frac{\widetilde{Q}_{1}^{k_{1}} \cdot \ldots \cdot \widetilde{Q}_{n}^{k_{n}}}{\widetilde{q}_{1}^{k_{1}+1} \cdot \ldots \cdot \widetilde{q}_{n}^{k_{n}+1}} d w= \\
=\left.\sum_{K \in \Re}(-1)^{\|K\|+n} \sum_{J} \frac{(-1)^{s(J)}}{\beta(K, J) !} \cdot \frac{\partial^{\|\beta(K, J)\|}}{\partial w^{\beta}(K, J)}\left[\widetilde{\Delta} \cdot w_{1}^{\gamma_{1}+1} \cdots w_{n}^{\gamma_{n}+1} \cdot \frac{\widetilde{Q}^{K}}{\widetilde{q}^{K+I}(J)}\right]\right|_{w=a_{J}} .
\end{gathered}
$$

Consider the following system of equations in two complex variables:

$$
\left\{\begin{array}{l}
f_{1}\left(z_{1}, z_{2}\right)=\left(1-a_{2} z_{2}\right)^{2}+a_{3} z_{1} z_{2}^{2}=0 \\
f_{2}\left(z_{1}, z_{2}\right)=\left(1-b_{1} z_{1}\right)^{2}\left(1-b_{2} z_{2}\right)+b_{3} z_{1}^{2} z_{2}=0 .
\end{array}\right.
$$

Then $Q_{m}, m=1,2$ have the form (20). The system (25) has, as is easy to verify, 5 roots $\left(z_{j 1}, z_{j 2}\right)$, $j=1,2,3,4,5$. If $a_{2} \neq b_{2}$, then these roots do not lie on the coordinate planes.

Let us change the variables $z_{1}=\frac{1}{w_{1}}, z_{2}=\frac{1}{w_{2}}$. Our system will take the form

$$
\left\{\begin{array}{l}
\tilde{f}_{1}=w_{1}\left(w_{2}-a_{2}\right)^{2}+a_{3}=0, \\
\widetilde{f}_{2}=\left(w_{1}-b_{1}\right)^{2}\left(w_{2}-b_{2}\right)+b_{3}=0 .
\end{array} .\right.
$$

Jacobian of the system (26)

$$
\widetilde{\Delta}=\left|\begin{array}{cc}
\left(w_{2}-a_{2}\right)^{2} & 2 w_{1}\left(w_{2}-a_{2}\right) \\
2\left(w_{1}-b_{1}\right)\left(w_{2}-b_{2}\right) & \left(w_{1}-b_{1}\right)^{2}
\end{array}\right|=
$$




$$
=\left(w_{1}-b_{1}\right)^{2}\left(w_{2}-a_{2}\right)^{2}-4 w_{1}\left(w_{1}-b_{1}\right)\left(w_{2}-a_{2}\right)\left(w_{2}-b_{2}\right) .
$$

Then, by Theorem 5, we obtain

$$
\begin{gathered}
\sigma_{\gamma}=\sum_{j=1}^{5} \frac{1}{z_{j 1}^{\gamma_{1}+1}} \cdot \frac{1}{z_{j 2}^{\gamma_{2}+1}}=\sum_{J} \sum_{K \in \Re} \frac{(-1)^{\|K\|+s(j)}}{(2 \pi i)^{2}} \times \\
\times \int_{\widetilde{\Gamma}_{h, a_{J}}} \frac{w_{1}^{\gamma_{1}+1} \cdot w_{2}^{\gamma_{2}+1} \cdot a_{3}^{k_{1}} \cdot b_{3}^{k_{2}} \cdot \widetilde{\Delta} \cdot d w_{1} \wedge d w_{2}}{w_{1}^{k_{1}+1}\left(w_{2}-a_{2}\right)^{2\left(k_{1}+1\right)} \cdot\left(w_{1}-b_{1}\right)^{2\left(k_{2}+1\right)}\left(w_{2}-b_{2}\right)^{k_{2}+1}} .
\end{gathered}
$$

Here the multi-indices $\Re=\left\{K=\left(k_{1}, k_{2}\right) \mid \exists m: \gamma_{m}+2>k_{1}+k_{2}, \quad m=1,2\right\}$. The cycles $\widetilde{\Gamma}_{h, a_{J}}$ are cycles of the form $\left\{\left|w_{1}\right|=r_{11},\left|w_{2}-b_{2}\right|=r_{22}\right\}$, taken with positive orientation, and $\left\{\left|w_{2}-a_{2}\right|=r_{12},\left|w_{1}-b_{1}\right|=r_{21}\right\}$ are with negative orientation.

In particular, calculating $J_{(0,0)}$, after some transformations we obtain

$$
\sigma_{(1,1)}=4 a_{2} b_{1}-\frac{a_{3} b_{2}}{\left(b_{2}-a_{2}\right)^{2}}
$$

without finding the roots.

Consider a system of equations in three complex variables:

$$
\left\{\begin{array}{l}
f_{1}\left(z_{1}, z_{2}, z_{3}\right)=1-a_{1} z_{1}-a_{2} z_{2}-a_{3} z_{3}+a_{1} a_{2} z_{1} z_{2}+a_{1} a_{3} z_{1} z_{3}+a_{2} a_{3} z_{2} z_{3}= \\
=\left(1-a_{1} z_{1}\right)\left(1-a_{2} z_{2}\right)\left(1-a_{3} z_{3}\right)+a_{1} a_{2} a_{3} z_{1} z_{2} z_{3}=0 \\
f_{2}\left(z_{1}, z_{2}, z_{3}\right)=1-b_{1} z_{1}-b_{2} z_{2}-b_{3} z_{3}+b_{1} b_{2} z_{1} z_{2}+b_{1} b_{3} z_{1} z_{3}+b_{2} b_{3} z_{2} z_{3}= \\
=\left(1-b_{1} z_{1}\right)\left(1-b_{2} z_{2}\right)\left(1-b_{3} z_{3}\right)+b_{1} b_{2} b_{3} z_{1} z_{2} z_{3}=0 \\
f_{3}\left(z_{1}, z_{2}, z_{3}\right)=1-c_{1} z_{1}-c_{2} z_{2}-c_{3} z_{3}+c_{1} c_{2} z_{1} z_{2}+c_{1} c_{3} z_{1} z_{3}+c_{2} c_{3} z_{2} z_{3}= \\
=\left(1-c_{1} z_{1}\right)\left(1-c_{2} z_{2}\right)\left(1-c_{3} z_{3}\right)+c_{1} c_{2} c_{3} z_{1} z_{2} z_{3}=0 .
\end{array} .\right.
$$

The roots of the system $(29)$ are $\left(z_{j 1}, z_{j 2}, z_{j 3}\right), j=1, \ldots, 12$.

Change the variables $z_{1}=\frac{1}{w_{1}}, z_{2}=\frac{1}{w_{2}}$ and $z_{3}=\frac{1}{w_{3}}$. Our system will take the form

$$
\left\{\begin{array}{l}
\tilde{f}_{1}=w_{1} w_{2} w_{3}-a_{1} w_{2} w_{3}-a_{2} w_{1} w_{3}-a_{3} w_{1} w_{2}+a_{1} a_{2} w_{3}+a_{1} a_{3} w_{2}+a_{2} a_{3} w_{1}= \\
=\left(w_{1}-a_{1}\right)\left(w_{2}-a_{2}\right)\left(w_{3}-a_{3}\right)+a_{1} a_{2} a_{3}=0, \\
\tilde{f}_{2}=w_{1} w_{2} w_{3}-b_{1} w_{2} w_{3}-b_{2} w_{1} w_{3}-b_{3} w_{1} w_{2}+b_{1} b_{2} w_{3}+b_{1} b_{3} w_{2}+b_{2} b_{3} w_{1}= \\
=\left(w_{1}-b_{1}\right)\left(w_{2}-b_{2}\right)\left(w_{3}-b_{3}\right)+b_{1} b_{2} b_{3}=0 \\
\tilde{f}_{3}=w_{1} w_{2} w_{3}-c_{1} w_{2} w_{3}-c_{2} w_{1} w_{3}-c_{3} w_{1} w_{2}+c_{1} c_{2} w_{3}+c_{1} c_{3} w_{2}+c_{2} c_{3} w_{1}= \\
=\left(w_{1}-c_{1}\right)\left(w_{2}-c_{2}\right)\left(w_{3}-c_{3}\right)+c_{1} c_{2} c_{3}=0 .
\end{array}\right.
$$

The Jacobian of the system (30)

$$
\begin{aligned}
& \widetilde{\Delta}=\left(w_{2}-a_{2}\right)\left(w_{3}-a_{3}\right)\left[\left(w_{1}-b_{1}\right)\left(w_{3}-b_{3}\right)\left(w_{1}-c_{1}\right)\left(w_{2}-c_{2}\right)-\left(w_{1}-b_{1}\right)\left(w_{2}-b_{2}\right)\left(w_{1}-c_{1}\right)\left(w_{3}-c_{3}\right)\right]- \\
& -\left(w_{1}-a_{1}\right)\left(w_{3}-a_{3}\right)\left[\left(w_{2}-b_{2}\right)\left(w_{3}-b_{3}\right)\left(w_{1}-c_{1}\right)\left(w_{2}-c_{2}\right)-\left(w_{1}-b_{1}\right)\left(w_{2}-b_{2}\right)\left(w_{2}-c_{2}\right)\left(w_{3}-c_{3}\right)\right]+ \\
& +\left(w_{1}-a_{1}\right)\left(w_{2}-a_{2}\right)\left[\left(w_{2}-b_{2}\right)\left(w_{3}-b_{3}\right)\left(w_{1}-c_{1}\right)\left(w_{3}-c_{3}\right)-\left(w_{1}-b_{1}\right)\left(w_{3}-b_{3}\right)\left(w_{2}-c_{2}\right)\left(w_{3}-c_{3}\right)\right] .
\end{aligned}
$$

Then, by Theorem 5 , we obtain $J_{(0,0,0)}=\sum_{J}(-1)^{s}(J)$

$$
\sum_{\|k\|<2} \frac{(-1)^{\|k\|}}{(2 \pi i)^{2}} \int_{\widetilde{\Gamma}_{q, a_{J}}} \frac{w_{1} w_{2} w_{3} \cdot\left(a_{1} a_{2} a_{3}\right)^{k_{1}}\left(b_{1} b_{2} b_{3}\right)^{k_{2}}\left(c_{1} c_{2} c_{3}\right)^{k_{3}} \cdot \widetilde{\Delta}}{\left(w_{1}-a_{1}\right)^{k_{1}+1}\left(w_{2}-a_{2}\right)^{k_{1}+1}\left(w_{3}-a_{3}\right)^{k_{1}+1}} \times
$$




$$
\times \frac{d w_{1} \wedge d w_{2} \wedge d w_{3}}{\left(w_{1}-b_{1}\right)^{k_{2}+1}\left(w_{2}-b_{2}\right)^{k_{2}+1}\left(w_{3}-b_{3}\right)^{k_{2}+1} \cdot\left(w_{1}-c_{1}\right)^{k_{3}+1}\left(w_{2}-c_{2}\right)^{k_{3}+1}\left(w_{3}-c_{3}\right)^{k_{3}+1}},
$$

where $\widetilde{\Gamma}_{q, a_{J}}$ are cycles of the form $\left\{\left|w_{1}-a_{1}\right|=r_{11},\left|w_{2}-b_{2}\right|=r_{22},\left|w_{3}-c_{3}\right|=r_{33}\right\} ;\left\{\left|w_{3}-a_{3}\right|=\right.$ $\left.r_{13},\left|w_{1}-b_{1}\right|=r_{21},\left|w_{2}-c_{2}\right|=r_{32}\right\} ;\left\{\left|w_{2}-a_{2}\right|=r_{12},\left|w_{3}-b_{3}\right|=r_{23},\left|w_{1}-c_{1}\right|=r_{31}\right\}$, taken with a positive orientation, and $\left\{\left|w_{1}-a_{1}\right|=r_{11},\left|w_{3}-b_{3}\right|=r_{23},\left|w_{2}-c_{2}\right|=r_{32}\right\}$; $\left\{\left|w_{2}-a_{2}\right|=r_{12},\left|w_{1}-b_{1}\right|=r_{21},\left|w_{3}-c_{3}\right|=r_{33}\right\} ;\left\{\left|w_{3}-a_{3}\right|=r_{13},\left|w_{2}-b_{2}\right|=r_{22},\left|w_{1}-c_{1}\right|=r_{31}\right\}$ with negative orientation.

Calculating these integrals, we get

$$
\begin{gathered}
-\sigma_{(1,1,1)}=J_{(0,0,0)}=a_{1} b_{2} c_{3}+a_{1} b_{3} c_{2}+a_{2} b_{1} c_{3}+a_{2} b_{3} c_{1}+a_{3} b_{1} c_{2}+a_{3} b_{2} c_{1}+ \\
+\frac{a_{3} c_{1} c_{2} c_{3}}{a_{3}-c_{3}} \cdot\left[\frac{b_{1}}{b_{1}-c_{1}}+\frac{b_{2}}{b_{2}-c_{2}}\right]+\frac{a_{1} b_{1} b_{2} b_{3}}{a_{1}-b_{1}} \cdot\left[\frac{c_{3}}{c_{3}-b_{3}}+\frac{c_{2}}{c_{2}-b_{2}}\right]+ \\
+\frac{a_{2} b_{1} b_{2} b_{3}}{a_{2}-b_{2}} \cdot\left[\frac{c_{3}}{c_{3}-b_{3}}+\frac{c_{1}}{c_{1}-b_{1}}\right]+\frac{a_{3} b_{1} b_{2} b_{3}}{a_{3}-b_{3}} \cdot\left[\frac{c_{2}}{c_{2}-b_{2}}+\frac{c_{1}}{c_{1}-b_{1}}\right]+ \\
+\frac{a_{1} c_{1}}{a_{1}-c_{1}} \cdot\left[\frac{b_{2} c_{2} c_{3}}{b_{2}-c_{2}}+\frac{b_{3} c_{2} c_{3}}{b_{3}-c_{3}}+\frac{a_{2} a_{3} b_{2}}{a_{2}-b_{2}}+\frac{a_{2} a_{3} b_{3}}{a_{3}-b_{3}}\right]+ \\
+\frac{a_{2} c_{2}}{a_{2}-c_{2}} \cdot\left[\frac{b_{1} c_{1} c_{3}}{b_{1}-c_{1}}+\frac{b_{3} c_{1} c_{3}}{b_{3}-c_{3}}+\frac{a_{1} a_{3} b_{3}}{a_{3}-b_{3}}+\frac{a_{1} a_{3} b_{1}}{a_{1}-b_{1}}\right] .
\end{gathered}
$$

So, we found the sums of the roots $\sigma_{(1,1,1)}$ without calculating the roots of the system themselves.

\section{General systems of transcendental equations}

Let $f_{1}(z), \ldots, f_{n}(z)$ be a system of functions holomorphic in a neighborhood of the origin in the multidimensional complex space $\mathbb{C}^{n}, z=\left(z_{1}, \ldots, z_{n}\right)$.

We expand the functions $f_{1}(z), \ldots, f_{n}(z)$ in Taylor series in the vicinity of the origin and consider a system of equations of the form

$$
f_{j}(z)=P_{j}(z)+Q_{j}(z)=0, \quad i=1, \ldots, n,
$$

where $P_{j}$ is the lowest homogeneous part of the Taylor expansion of the function $f_{j}(z)$. The degree of all monomials (with respect to the totality of variables) included in $P_{j}$, is equal to $m_{j}$, $j=1, \ldots, n$. In the functions $Q_{j} l$, the degrees of all monomials are strictly greater $\operatorname{than} m_{j}$.

The expansion of the functions $Q_{j}, P_{j}, j=1, \ldots, n$ in a neighborhood of zero in Taylor series converging absolutely and uniformly in this neighborhood has the form

$$
\begin{aligned}
Q_{j}(z) & =\sum_{\|\alpha\|>m_{j}} a_{\alpha}^{j} z^{\alpha}, \\
P_{j}(z) & =\sum_{\|\beta\|=m_{j}} b_{\beta}^{j} z^{\beta}, \\
j & =1, \ldots, n,
\end{aligned}
$$

where $\alpha=\left(\alpha_{1}, \ldots, \alpha_{n}\right), \beta=\left(\beta_{1}, \ldots, \beta_{n}\right)$ are multi-indexes, i.e. $\alpha_{j}$ and $\beta_{j}$ are non-negative integers, $j=1, \ldots, n,\|\alpha\|=\alpha_{1}+\ldots+\alpha_{n},\|\beta\|=\beta_{1}+\ldots+\beta_{n}$, and monomials $z^{\alpha}=z_{1}^{\alpha_{1}}$. $z_{2}^{\alpha_{2}} \cdots z_{n}^{\alpha_{n}}, z^{\beta}=z_{1}^{\beta_{1}} \cdot z_{2}^{\beta_{2}} \cdots z_{n}^{\beta_{n}}$.

In what follows, we will assume that the system of polynomials $P_{1}(z), \ldots, P_{n}(z)$ is nondegenerate, that is, its common zero is only point 0 , the origin. 
Consider an open set (a special analytic polyhedron) of the form

$$
D_{P}\left(r_{1}, \ldots, r_{n}\right)=\left\{z:\left|P_{j}(z)\right|<r_{j}, i=j, \ldots, n\right\},
$$

where $r_{1}, \ldots, r_{n}$ are positive numbers. Its skeleton has the form

$$
\Gamma_{P}\left(r_{1}, \ldots, r_{n}\right)=\Gamma_{P}(r)=\left\{z:\left|P_{j}(z)\right|=r_{j}, j=1, \ldots, n\right\} .
$$

Let us start with a statement.

Lemma 3. The next equality is true

$$
\begin{gathered}
J_{\gamma}=\frac{1}{(2 \pi i)^{n}} \int_{\Gamma_{P}} \frac{1}{z_{1}^{\gamma_{1}+1} \cdot z_{2}^{\gamma_{2}+1} \ldots z_{n}^{\gamma_{n}+1}} \cdot \frac{d f_{1}}{f_{1}} \wedge \frac{d f_{2}}{f_{2}} \wedge \ldots \wedge \frac{d f_{n}}{f_{n}}= \\
=\frac{(-1)^{n}}{(2 \pi i)^{n}} \int_{\Gamma_{\tilde{P}}} w_{1}^{\gamma_{1}+1} \cdot w_{2}^{\gamma_{2}+1} \cdots w_{n}^{\gamma_{n}+1} \cdot \frac{d \tilde{f}_{1}}{\tilde{f}_{1}} \wedge \frac{d \tilde{f}_{2}}{\tilde{f}_{2}} \wedge \ldots \wedge \frac{d \tilde{f}_{n}}{\tilde{f}_{n}}=(-1)^{n} \tilde{J}_{\gamma} .
\end{gathered}
$$

For what follows, we need a generalized formula for transforming the Grothendieck residue.

Theorem 6. Let $h(w)$ be a holomorphic function, and the polynomials $f_{k}(w)$ and $g_{j}(w)$, $j, k=1, \ldots, n$, are related by the relations

$$
g_{j}=\sum_{k=1}^{n} a_{j k} f_{k}, \quad j=1,2, \ldots, n,
$$

the matrix $A=\left\|a_{j k}\right\|_{j, k=1}^{n}$ consists of polynomials. Consider the cycles

$$
\begin{gathered}
\Gamma_{f}=\left\{w:\left|f_{j}(w)\right|=r_{j}, j=1, \ldots, n\right\}, \\
\Gamma_{g}=\left\{w:\left|g_{j}(z)\right|=r_{j}, j=1, \ldots, n\right\},
\end{gathered}
$$

where all $r_{j}>0$. Then the equality

$$
\int_{\Gamma_{f}} h(w) \frac{d w}{f^{\alpha}}=\sum_{K, \sum_{s=1}^{n} k_{s j}=\beta_{s}} \frac{\beta !}{\prod_{s, j=1}^{n}\left(k_{s j}\right) !} \int_{\Gamma_{g}} h(w) \frac{\operatorname{det} A \prod_{s, j=1}^{n} a_{s j}^{k_{s j}} d w}{g^{\beta}}
$$

holds. Here $\beta !=\beta_{1} ! \beta_{2} ! \ldots \beta_{n}, \beta=\left(\beta_{1}, \beta_{2}, \ldots, \beta_{n}\right)$, the summation in the formula is over all non-negative integer matrices $K=\left\|k_{s j}\right\|_{s, j=1}^{n}$ with the conditions that the sum $\sum_{s=1}^{n} k_{s j}=\alpha_{j}$, then $\beta_{j}=\sum_{j=1}^{n} k_{j s}$. Heref ${ }^{\alpha}=f_{1}^{\alpha_{1}} \cdots f_{n}^{\alpha_{n}}, g^{\beta}=g_{1}^{\beta_{1}} \cdots g_{n}^{\beta_{n}}$.

Theorem 7. The next formulas are valid

$$
\begin{gathered}
\sum_{j=1}^{p} \frac{1}{z_{j 1}^{\gamma_{1}+1} \cdot z_{j 2}^{\gamma_{2}+1} \cdots z_{j n}^{\gamma_{n}+1}}= \\
\left.=(2 \pi i)^{n}\right) \int_{\Gamma_{\tilde{P}}} w_{1}^{\gamma_{1}+1} \cdot w_{2}^{\gamma_{2}+1} \cdots w_{n}^{\gamma_{n}+1} \cdot \frac{d \tilde{f}_{1}}{\tilde{f}_{1}} \wedge \frac{d \tilde{f}_{2}}{\tilde{f}_{2}} \wedge \ldots \wedge \frac{d \tilde{f}_{n}}{\tilde{f}_{n}}=
\end{gathered}
$$




$$
\begin{gathered}
=\sum_{\|\alpha\| \leqslant\|\gamma\|+n} \frac{(-1)^{n+\|\alpha\|}}{(2 \pi i)^{n}} \int_{\Gamma_{\tilde{P}}} w_{1}^{\gamma_{1}+1} \cdot w_{2}^{\gamma_{2}+1} \cdots w_{n}^{\gamma_{n}+1} \times \\
\times \frac{\tilde{\Delta} \cdot \tilde{Q}_{1}^{\alpha_{1}} \cdot \tilde{Q}_{2}^{\alpha_{2}} \cdots \tilde{Q}_{n}^{\alpha_{n}} d w_{1} \wedge d w_{2} \wedge \ldots \wedge d w_{n}}{\tilde{P}_{1}^{\alpha_{1}+1} \cdot \tilde{P}_{2}^{\alpha_{2}+1} \cdots \tilde{P}_{n}^{\alpha_{n}+1}}= \\
=\sum_{\|K\| \leqslant\|\gamma\|+n} \frac{(-1)^{\|K\|+n} \prod_{s=1}^{n}\left(\sum_{j=1}^{n} k_{s j}\right) !}{\prod_{s, j=1}^{n}\left(k_{s j}\right) !}\left[\frac{w^{\gamma+I} \cdot \tilde{\Delta} \cdot \operatorname{det} A \cdot Q^{\alpha} \prod_{s, j=1}^{n} a_{s j}^{k_{s j}}}{\prod_{j=1}^{n} w_{j}^{\beta_{j} N_{j}+\beta_{j}+N_{j}}}\right],
\end{gathered}
$$

where $\|K\|=\sum_{s, j=1}^{n} k_{s j}$, and the functional $\mathfrak{M}$ assigns its free term to the Laurent polynomial.

In fact, in Theorem 7, analogs of the classical Waring formulas for finding power sums of roots of a system of algebraic equations are obtained.

Consider a system of equations in two complex variables

$$
\left\{\begin{array}{l}
f_{1}\left(z_{1}, z_{2}\right)=a_{1} z_{1}-a_{2} z_{2}+z_{1}^{2}=0 \\
f_{2}\left(z_{1}, z_{2}\right)=b_{1} z_{1}+b_{2} z_{2}+z_{2}^{2}=0 .
\end{array}\right.
$$

It satisfies the conditions on $Q_{j}(z)$ We will assume that $a_{1} b_{2}+a_{2} b_{1} \neq 0$, i.e. the system of lower homogeneous polynomials is nondegenerate.

Let us change variables $z_{1}=\frac{1}{w_{1}}, z_{2}=\frac{1}{w_{2}}$. Our system will take the form

$$
\left\{\begin{array}{l}
\tilde{f}_{1}=-a_{2} w_{1}^{2}+a_{1} w_{1} w_{2}+w_{2}=0, \\
\tilde{f}_{2}=b_{2} w_{1} w_{2}+b_{1} w_{2}^{2}+w_{1}=0 .
\end{array} .\right.
$$

This system has 4 roots, on the coordinate planes there is one root - $(0,0)$.

The Jacobian of the system (38)

$$
\begin{gathered}
\tilde{\Delta}=\left|\begin{array}{cc}
-2 a_{2} w_{1}+a_{1} w_{2} & a_{1} w_{1}+1 \\
b_{2} w_{2}+1 & 2 b_{1} w_{2}+b_{2} w_{1}
\end{array}\right|= \\
=-2 a_{2} b_{2} w_{1}^{2}-4 a_{2} b_{1} w_{1} w_{2}+2 a_{1} b_{1} w_{2}^{2}-a_{1} w_{1}-b_{2} w_{2}-1 .
\end{gathered}
$$

Notice that

$$
\begin{gathered}
\tilde{Q}_{1}=w_{2}, \quad \tilde{Q}_{2}=w_{1} . \\
\tilde{P}_{1}=-a_{2} w_{1}^{2}+a_{1} w_{1} w_{2}, \quad \tilde{P}_{2}=b_{2} w_{1} w_{2}+b_{1} w_{2}^{2} .
\end{gathered}
$$

To find the matrix $A$ we use Example 8.3 from [4].

We introduce the matrix

$$
\operatorname{Res}=\left(\begin{array}{cccc}
-a_{2} & a_{1} & 0 & 0 \\
0 & -a_{2} & a_{1} & 0 \\
0 & b_{2} & b_{1} & 0 \\
0 & 0 & b_{2} & b_{1}
\end{array}\right)
$$


The determinant $\Delta$ of the matrix Res is $\Delta=a_{2} b_{1}\left(a_{2} b_{1}+a_{1} b_{2}\right)$. Let us calculate some minors according to example 8.3 from [4]:

$$
\begin{array}{lll}
\tilde{\Delta}_{1}=\left|\begin{array}{ccc}
-a_{2} & a_{1} & 0 \\
b_{2} & b_{1} & 0 \\
0 & b_{2} & b_{1}
\end{array}\right|=-a_{2} b_{1}^{2}-a_{1} b_{1} b_{2}, & \tilde{\Delta}_{2}=-\left|\begin{array}{ccc}
a_{1} & 0 & 0 \\
b_{2} & b_{1} & 0 \\
0 & b_{2} & b_{1}
\end{array}\right|=-a_{1} b_{1}^{2}, \\
\widetilde{\Delta}_{3}=\left|\begin{array}{ccc}
a_{1} & 0 & 0 \\
-a_{2} & a_{1} & 0 \\
0 & b_{2} & b_{1}
\end{array}\right|=a_{1}^{2} b_{1}, & \tilde{\Delta}_{4}=-\left|\begin{array}{ccc}
a_{1} & 0 & 0 \\
-a_{2} & a_{1} & 0 \\
b_{2} & b_{1} & 0
\end{array}\right|=0 . \\
\Delta_{1}=-\left|\begin{array}{ccc}
0 & -a_{2} & a_{1} \\
0 & b_{2} & b_{1} \\
0 & 0 & b_{2}
\end{array}\right|=0, & \Delta_{2}=\left|\begin{array}{ccc}
-a_{2} & a_{1} & 0 \\
0 & b_{2} & b_{1} \\
0 & 0 & b_{2}
\end{array}\right|=-a_{2} b_{2}^{2}, \\
\Delta_{3}=-\left|\begin{array}{ccc}
-a_{2} & a_{1} & 0 \\
0 & -a_{2} & a_{1} \\
0 & 0 & b_{2}
\end{array}\right|=-a_{2}^{2} b_{2}, & \Delta_{4}=\left|\begin{array}{ccc}
-a_{2} & a_{1} & 0 \\
0 & -a_{2} & a_{1} \\
0 & b_{2} & b_{1}
\end{array}\right|=a_{2}^{2} b_{1}+a_{1} a_{2} b_{2} .
\end{array}
$$

Therefore, the elements $a_{i j}$ of the matrix $A$ are

$$
\begin{gathered}
a_{11}=\frac{1}{\Delta}\left(\tilde{\Delta}_{1} w_{1}+\tilde{\Delta}_{2} w_{2}\right)=\frac{1}{\Delta}\left(\left(-a_{2} b_{1}^{2}-a_{1} b_{1} b_{2}\right) w_{1}-a_{1} b_{1}^{2} w_{2}\right), \\
a_{12}=\frac{1}{\Delta}\left(\widetilde{\Delta}_{3} w_{1}+\widetilde{\Delta}_{4} w_{2}\right)=\frac{a_{1}^{2} b_{1} w_{1}}{\Delta}, \quad a_{21}=\frac{1}{\Delta}\left(\Delta_{1} w_{1}+\Delta_{2} w_{2}\right)=\frac{-a_{2} b_{2}^{2} w_{2}}{\Delta}, \\
a_{22}=\frac{1}{\Delta}\left(\Delta_{3} w_{1}+\Delta_{4} w_{2}\right)=\frac{1}{\Delta}\left(-a_{2}^{2} b_{2} w_{1}+\left(a_{2}^{2} b_{1}+a_{1} a_{2} b_{2}\right) w_{2}\right) .
\end{gathered}
$$

Then it is easy to check that

$$
w_{1}^{3}=a_{11} \tilde{P}_{1}+a_{12} \tilde{P}_{2}, \quad w_{2}^{3}=a_{21} \tilde{P}_{1}+a_{22} \tilde{P}_{2} .
$$

We calculate $\operatorname{det} A$ :

$$
\operatorname{det} A=\frac{1}{\Delta}\left(a_{2} b_{2} w_{1}^{2}-a_{2} b_{1} w_{1} w_{2}-a_{1} b_{1} w_{2}^{2}\right) .
$$

By Theorem 7

$$
\begin{gathered}
J_{(0,0)}=\sum_{\|K\| \leqslant 2} \frac{(-1)^{\|K\|} \cdot\left(k_{11}+k_{12}\right) ! \cdot\left(k_{21}+k_{22}\right) !}{k_{11} ! \cdot k_{12} ! \cdot k_{21} ! \cdot k_{22} !} \times \\
\times \mathfrak{M}\left[\frac{\tilde{\Delta} \cdot \operatorname{det} A \cdot \tilde{Q}_{1}^{k_{11}+k_{21}} \cdot \tilde{Q}_{2}^{k_{12}+k_{22}} \cdot a_{11}^{k_{11}} \cdot a_{12}^{k_{12}} \cdot a_{21}^{k_{21}} \cdot a_{22}^{k_{22}}}{w_{1}^{3\left(k_{11}+k_{12}\right)+1} \cdot w_{2}^{3\left(k_{21}+k_{22}\right)+1}}\right] .
\end{gathered}
$$

We denote $\bar{\Delta}=a_{2} b_{1}+a_{1} b_{2}$. Cumbersome but simple calculations (using the definition of the functional $\mathfrak{M}$ ) give that

$$
\begin{gathered}
J_{(0,0)}=\frac{1}{\bar{\Delta}}-\frac{2 a_{1} b_{2}}{a_{2} b_{1} \bar{\Delta}}+\frac{6 a_{1}^{2} b_{2}^{2}}{a_{2} b_{1} \bar{\Delta}^{2}}-\frac{b_{2}^{3}}{b_{1} \bar{\Delta}^{2}}+\frac{a_{1}^{3}}{a_{2} \bar{\Delta}^{2}}+\frac{8 a_{1} b_{2}}{\bar{\Delta}^{2}}-\frac{4}{a_{2} b_{1}}= \\
=\frac{a_{1}^{3}}{a_{2} \bar{\Delta}^{2}}-\frac{a_{1} b_{2}}{\bar{\Delta}^{2}}-\frac{3 a_{2} b_{1}}{\bar{\Delta}^{2}}-\frac{b_{2}^{3}}{b_{1} \bar{\Delta}^{2}} .
\end{gathered}
$$

This work was supported by the Russian Science Foundation, grant Complex analytic geometry and multidimensional deductions. Number: 20-11-2011\%. 


\section{References}

[1] L.A.Aizenberg, On a formula of the gereralized multidimensional logarithmic residue and the solution of system of nonlinear equations, Sov. Math. Doc., 18(1977), 691-695.

[2] L.A.Aizenberg, A.P.Yuzhakov, Integral representations and residues in multidimensional complex analysis, Amer. Math. Monographs, AMS, Providence, 1983.

[3] A.K.Tsikh, Multidimensional residues and their applications. Translations of Mathematical Monographs, American Mathematical Society, Providence, RI, 1992.

[4] V.I.Bykov, A.M.Kytmanov, M.Z.Lazman, Elimination Methods in Polynomial Computer Algebra, Springer, New-York, 1998.

[5] L.A.Aizenberg, A.M.Kytmanov, Multidimensional analogues of Newton's formulas for systems of nonlinear algebraic equations and some of their applications, Siberian Mathematical Journal, 22(1981), 180-189.

[6] V.I.Bykov, Modeling of the critical phenomena in chemical kinetics, Komkniga, Moscow, 2006 (in Russian).

[7] V.I.Bykov, S.B.Tsybenova, Non-linear models of chemical kinetics, KRASAND, Moscow, 2011 (in Russian).

[8] A.M.Kytmanov, Z.E.Potapova Formulas for determining power sums of roots of systems of meromorphic functions, Izvestiya VUZ. Matematika, 49(2005), no. 8, 36-45 (in Russian).

[9] V.I.Bykov, A.M.Kytmanov, S.G.Myslivets, Power sums of nonlinear systems of equations, Dokl. Math., 76(2007), 641-644. DOI: 10.1134/S1064562407050018

[10] A.M.Kytmanov, E.K.Myshkina, Russian Mathematics, 57(2013), no. 12, 31-43. DOI: $10.3103 /$ S1066369X13120049

[11] O.V.Khodos, On Some Systems of Non-algebraic Equations in $\mathbb{C}^{n}$, Journal of Siberian Federal University. Mathematics \&Physics, 7(2014), no. 4, 455-465.

[12] A.M.Kytmanov, E.K.Myshkina, On calculation of power sums of roots for one class of systems of non-algebraic equations, Sib. Elektron. Mat. Rep., 12(2015), 190-209 (in Russian). DOI: $10.17377 /$ semi.2015.12.016

[13] A.A.Kytmanov, A.M.Kytmanov, E.K.Myshkina, Finding residue integrals for systems of non-algebraic equations in $\mathbb{C}^{n}$, Journal of Symbolic Computation, 66(2015), 98-110.

DOI: $10.1016 / \mathrm{j} . j s c .2014 .01 .007$

[14] A.V.Kytmanov, E.R.Myshkina, J. Math. Sciences, 213(2016), no. 6, 868-886. DOI: $10.1007 / \mathrm{s} 10958-016-2748-7$

[15] A.M.Kytmanov, O.V.Khodos, On systems of non-algebraic equation in $\mathbb{C}^{n}$, Contemporary Mathematics, 662(2016), 77-88.

[16] A.M.Kytmanov, O.V.Khodos, On localization of the zeros of an entire function of finite order of growth, Journal Complex Analysis and Operator Theory, 11(2017), no. 2, 393-416.

[17] A.M.Kytmanov, Ya.M.Naprienko, One approach to finding the resultant of two entire functions, Complex variables and elliptic equations 62 (2) (2017), 269-286. 
[18] A.M.Kytmanov, O.V.Khodos, On one approach to determining the resultant of two entire functions, Russian Math., no. 4, 2018, 49-59.

[19] A.A.Kytmanov, A.M.Kytmanov, E.K.Myshkina, Residue Integrals and Waring's Formulas for a Class of Systems of Transcendental Equations in $\mathbb{C}^{n}$, Journal of Complex variables and Elliptic Equations, 64(2018), no. 1. 93-111. DOI: 10.1080/17476933.2017.1419210

[20] A.M.Kytmanov, E.K.Myshkina, Residue integrals and Waring formulas for algebraic and transcendental systems of equations, Russian Math., 2019, no. 5, 40-55.

DOI: $10.26907 / 0021-3446-2019-5-40-55$

[21] A.M.Kytmanov, Algebraic and transcendental systems of equations, SFU, Krasnoyarsk, 2019 .

[22] M.Passare, A.Tsikh, Residue integrals and their Mellin transforms, Can. J. Math., 47(1995), no. $5,1037-1050$.

\title{
О трансцендентных системах уравнений
} Александр М. Кытманов
Ольга В. Ходос

Сибирский федеральный университет Красноярск, Российская Федерация

\begin{abstract}
Аннотация. Рассмотрены различные типы систем трансцендентных уравнений: простейшие, специальные и общие. Поскольку число корней таких систем, как правило, бесконечно, то необходимо изучить степенные суммы корней в отрицательной степени. Получены формулы для нахождения вычетных интегралов, их связь со степенными суммами корней в отрицательной степени, многомерные аналоги формул Варинга. Приведены различные примеры трансцендентных систем уравнений и вычислены суммы многомерных числовых рядов.
\end{abstract}

Ключевые слова: трансцендентные системы уравнений, степенные суммы корней, вычетные интегралы. 